\title{
Virtuality Supports Reality for e-Health Applications
}

\author{
Giovanni Saggio and Carlo A lberto Pinto \\ University of Rome "Tor V ergata"
}

Italy

\section{Introduction}

The "virtualization" process of the reality is unavoidable and cannot be arrested since it goes deeply towards the process of dematerialization initiate with the post-industrialization era. Formerly, in fact, the power and the richness were in the hands of people with large amounts of goods or lands, but nowadays are in the hands of people who sell intangible applications (software and firmware) or goods with poor material but highly rich contents (notebooks, mobile phones, ipods,..) or even only no matter services (telecom, web, educational,... companies).

Hence the "virtualization" is more and more becoming a so powerful tool that can have the capability to reproduce, augment and even overcome "reality".

In this view the "virtuality", joined to the know-how, is the successful key for new fundamental achievement in really many fields, but some in particular deserve special attention, i.e. the health-focused disciplines, so important because there are highly concentrated the monetary, political and social interests. In this context our aim is to furnish an overview on how nowadays the virtuality supports reality for e-health applications.

\section{Virtuality reproduces, augments and overcomes reality}

Even if the term "virtual" was born for unreal things, over the time it tends to be more and more used in reference to things that mimic their "real" equivalents, especially via pc applications. So we know of virtual library, virtual earth, virtual work, virtual museum, virtual tour, and so on.

We can refer the "real" mimed by the "virtual" as a "virtualization" process, which has now also the aim to enrich, enhance and, in some way, boost the possibilities offered by the "restricted" real world.

Limiting, for the moment, out attention to the mere aspect of reproduction of the reality, the "virtuality" expresses a virtual vision of the real world. Nowadays this process can be produced by interesting 3D techniques such as stereoscopy and holography.

Actually the stereoscopy was invented in the remote 1832 by Charles Wheathstone, but did not present any practical application till now. This technique exploits the human binocular vision to place virtual objects in a 3D space. An example is reproduced in Fig. 1 where users with special glasses have the impression of seeing "floating" planets in the room. The application in Fig. 1 has been developed by our collaborators of the PFM Multimedia Company (www.pfmmultimedia.it). 


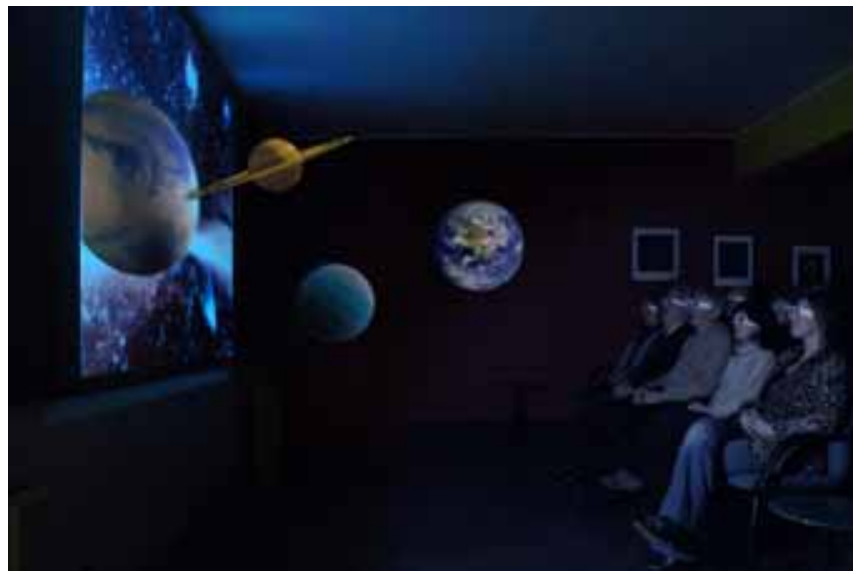

Fig. 1. Users donning special glasses see "floating" planets in the room (Courtesy by PFM Multimedia Company)

The holography was theorized for the first time in 1947 by Dennis Gabor and the first practical applications were possible only few years later by the adoption of the laser technology. One of the latest holography technique has been applied and can be appreciated in the site of Pompei (Naples, Italy) where in the ancient house of Polibio an hologram illustrates to the visitors the characteristics of the domus (see Fig. 2). This is a new technique indeed, being based on ultrasonic transducers which diffuse micro-particles of air mixed with nebulized water, as a support to the projection of the images. Again, this application has been developed by our collaborators of the PFM Multimedia Company.

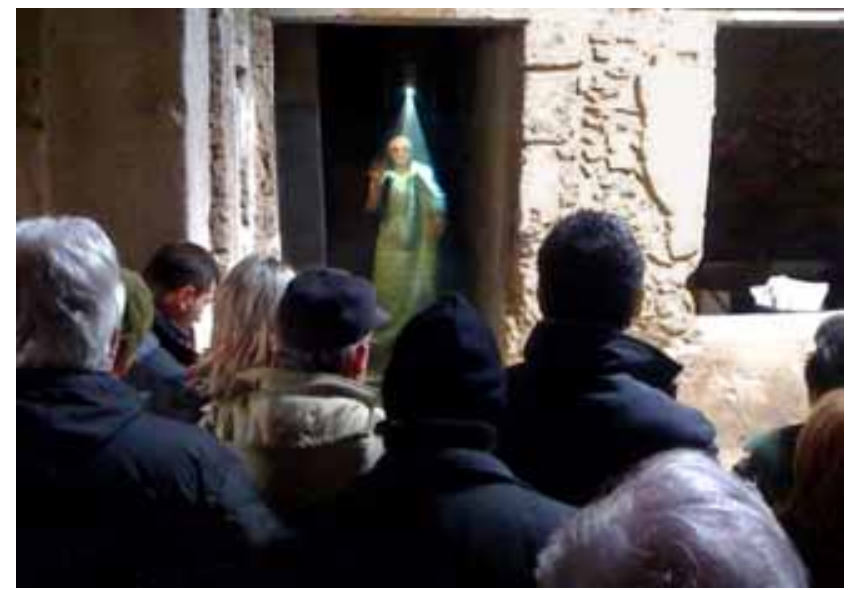

Fig. 2. A 3D holographic virtualization of Polibio in his domus in Pompei (Courtesy by PFM Multimedia Company)

The reported examples deal not only with the possibility to reproduce reality in a virtual world, but also demonstrate how virtuality "augments" reality, giving visibility to ancient no more existing world. 
A mong all the virtual pc applications, which "copy" the reality, one of the most interesting is more and more becoming the avatar reproduction of measured real human postures, possible thanks to the current availability of suitable sensors and electronic stuff.

In this view, we can refer to mere shoots of postures by cameras or webcams which can "see" the reality from a multi-point-of-view, but one at time. An example of this is represented by the known gait analysis system, which consists in the systematic study of human/ animal locomotion, augmented by instrumentation for measuring body movements, but mainly by means of multiple cameras view.

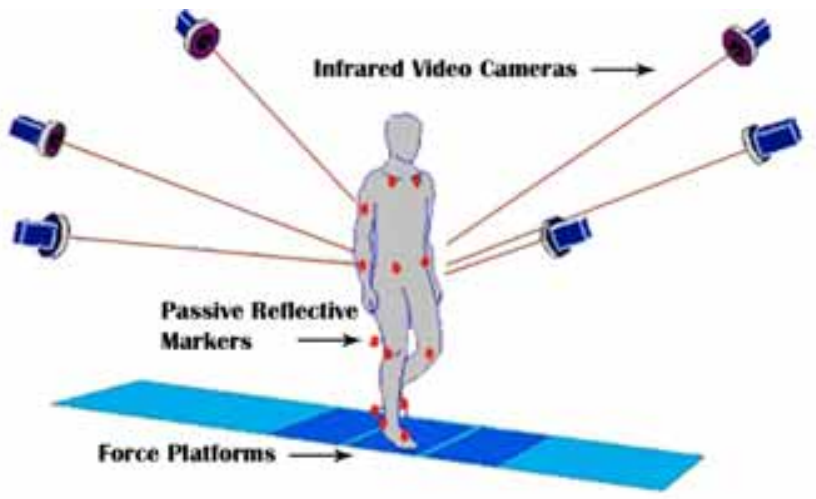

Fig. 3. A schematization of the camera based gait analysis system

But actually here we want to consider the new, more interesting and innovative, wearable sensorized systems which can accurately measure all the angles of human joints, pointing out finger, wrist, pelvis, neck, knee,.. movements, and spatial positions of arms, trunk, head, legs,.. The "wearability" of these new systems allow the advantage of unnecessity of a prepared scenario and have the capability to quantitatively measure the movements in every time and every space without limitations. The wearable systems make it possible to measure every single pose and movement of a person, so allowing the real time reproduction in Virtual Reality (VR), by means of complex avatars which can be incredibly similar to the original. We are here treating of new frontier systems capable to measure all human postures (or single parts of them) and to reproduce such postures in VR.

These systems have a potential huge number of applications, covering several different areas: working, sporting, gaming,.. with implications on social, military, medical, musical, edutainment, .. fields.

In the social area the measure and the subsequent "virtualization" of human postures can lead to a process capable to translate the sign language into single words and phrases, in home automation a real movement can be virtually recognized and an action can be associated to it, the virtualization of real acts can be very useful for improving the ergonomics of some stuff, etc.

In the work area the "virtualization" of real human actions can be useful for simulating dangerous activities, for verifying "off-line" the effect of some actions, for simulating the manipulation of hazardous stuff, for designing assistance, etc.

In the computer science area the "virtualized" reality can be useful for realizing new input interfaces or for implementing new automatic programming tools. In particular, within such frame, we realized a computer interaction system with no necessity of mouse and keyboard 
inputs, being the commands obtained by the "translation" of finger movements of the user (see Fig. 4).
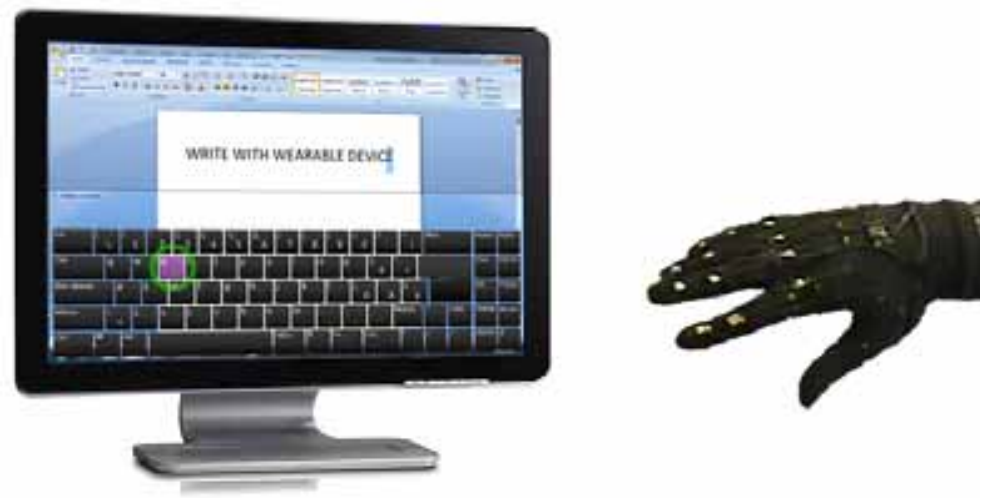

Fig. 4. Mouse and keyboard are unnecessary if computer interactions are made by a sensorized glove capable to accept commands obtained by finger motions.

In the music area the "virtualization" can reproduce real music instruments with good accuracy avoiding the expenses and spaces paid in reality, and the virtual instruments can be played simulating the action of the player or associating a single note/ chord to a real human body posture (Costantini et al., 2010).

Perhaps the army, the navy and the air forces are the more potentially interested in the "virtualization" process of human movements, since the necessity of simulating war scenarios, and the necessity of predicting the consequences of soldier acts in several potential environments.

So going ahead for all the other previous mentioned fields, "virtuality" can reproduce "reality" for the aims of training, educating, assisting, experiencing people.

But it is in the health-focused areas that the measure of real human postures, and the subsequent reproduction by means of pc avatars, can lead to the most fascinating and useful applications. In fact the virtualization process of patient's postures can be very useful in motor therapy, so that doctors can better identify pathologies, in rehabilitation, so to rightly evaluate pre-post effect of surgery, in functional analysis, so to create a database to classify the residual movements of the patient, and so on. On the other end not only the measure of patient's postures can be so useful, but even the doctor's ones! In fact, measuring the doctor's hand movements can be fundamental for surgical training or skills evaluation for virtual implementations of new procedures, for realizing minimal invasive surgery techniques, even for implementing tele-surgery in a way that the doctor's movements are faithfully remotely replayed by robot's arms.

In such a frame is the so called augmented reality which plays a winning role (Geisen, 2005). The concept of Augmented Reality, short AR (sometimes referred as Mixed Reality), comes from a fusion of digital data together with the human perception of the environment, so that the virtual objects overlay the reality on a pc screen (see Fig. 5). To the aim of upgrading performances and accuracies of the overall system and increasing the comfort for the user, we think here to the digital data obtained by means of the previously mentioned wearable systems. Augmented and Mixed Reality technology offer seamless visualization of text- 
based physiological data and various graphical 3D data onto the patient's body. Thanks to the wearable sensors together with the AR the user can see himself into the real world, with the virtual objects superimposed upon or composited with it. So AR supplements reality and, ideally, "it would appear to the user that the virtual and real objects coexisted in the same space." (Azuma, 1997)

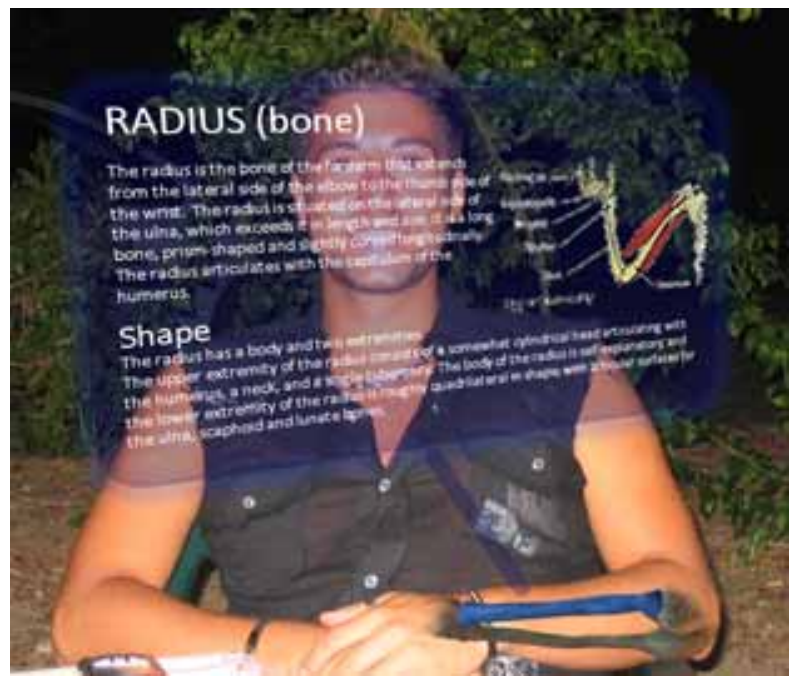

Fig. 5. An example of AR imagine. Information about bones are superimposed over the real picture

Potentials and limits of current AR in surgery have been reported elsewhere (Shuhaiber, 2004), so here our aim is not to discuss about that, but the introduction of novel possibilities which comes from different interfaces thanks to the wearable systems, not applied till now, which can even overcome the, for some aspect, still futuristic AR.

As al ready mentioned, the AR technology is both for real and for simulate surgery. An example of the latter comes from the ImmersiveTouch ${ }^{\mathrm{TM}}$-SENSIMMER ${ }^{\circledR}$ system (www.immersivetouch.com), which integrates a haptic device with a head and hand tracking system and a high resolution high pixel-density stereoscopic display. The haptic device is in some way "merged" with the high resolution 3D graphics, giving the user a more realistic and natural means to manipulateand modify 3D data in real time.

Since the key element in AR for surgery is becoming more and more the exact measure of doctor's hand postures, our argumentation will be especially focused on that in the following paragraphs.

The virtualization can be even enhanced and extended thanks to 3D visualisation-related knowledge. Examples come from the 3DVisA (3dvisa.cch.kcl.ac.uk), Altair4 Multimedia (www.altair4.it), PFM Multimedia (www.pfmmultimedia.it). So virtuality results a key element even for hidden, inaccessible or alternative reality.

The VR can boost the possibilities of the real world since it can even represents the hidden reality. Let's think, for instance, to the wearable sensorized system donned by soldiers: even if no camera pictures are possible, we can have in any case their postures real-time reproduces in a remote location, so to guarantee information otherwise impossible to obtain (see Fig. 6) 


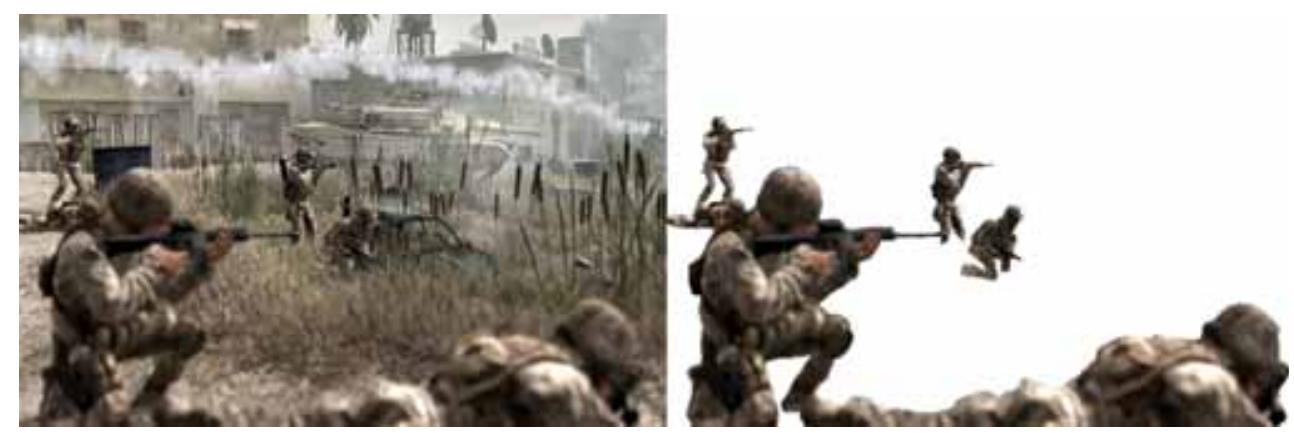

Fig. 6. The wearable sensorized system makes it possi ble to obtain fundamental posture data of soldiers even if no camera shootings are possible. On the left the real situation, on the right the visualization of the obtained information from the sensorized garments.

\subsection{From reality to virtuality}

To reproduce reality in a virtual environment, let's start analyzing the so called Virtual World and all its components. To transpose reality into a 3D scenario three key topics should be considered: M odels, Textures and Lights.

$M$ odelling is the art and science of creating a surface that mimics the shape of a real object. Each object of the virtual world has a shape and a size and this entity is called M esh. In meshes everything is built from three basic structures: $V$ ertices, Edges and Faces (see Fig. 7).

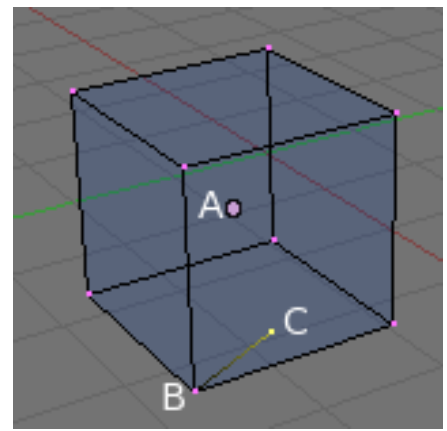

Fig. 7. Vertices, Edges and Faces: $C$ is a vertex, between $B$ and $C$ there is an edge and $A$ is the mesh center point

A vertex is primarily a single point or a position in a 3D space. A straight line connecting two vertices is an edge; this is the wire that is seen when a mesh is looked at in wireframe view. Vertices and edges are usually invisible on the rendered image.

Edges are used to construct faces which are the highest level structure in a mesh and are used to build the surface of the object. A face locates the area between either three or four vertices (respectively triangles and quadrangles), with an edge on every side. The mentioned mesh can be defined as a set of connected vertices and sometimes thousands of vertices are necessary to built complex objects.

It is possible to assemble the vertices in groups forming the so called V ertex Groups, so to reusing parts of a mesh for making copies and, eventually, hiding "everything else" while 
details are worked and so on. Vertex Groups identify sub-components of an object, like the joints of a hand or the hinges of a door. With vertex groups we can easily select and work on them in isolation without the necessity to create apart objects.

The "virtual world", how described till now, produces smooth, uniform objects that can be animated, but such objects are not yet particularly similar to the real counterpart, because uniformity tends to be uncommon and out of place. In order to approach a more realistic scenario, a 3D model Textures can be applied so to modify the reflectivity, specularity, roughness and other surface qualities of a material (see Fig. 8).

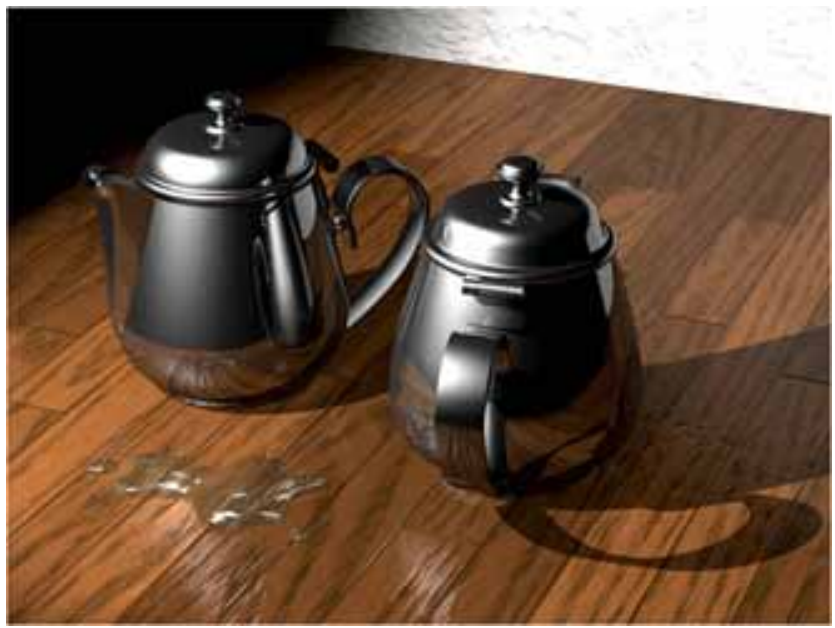

Fig. 8. This image is an example of results obtained from texture application

The third issue, Lighting, is a very important topic in rendering, standing equal to models and textures. A simple model can become very realistic if a light source is skillfully adopted, while without a proper lighting scheme the most accurately modeled and textured scene will yield poor results. We have to apply the same "lighting-rules" as in the real world, which is never lit by a single light source, indeed even if a single light is present, its rays can bounce off objects being reirradiated all over the scene. In this way every single part of the image or 3D space is softly shadowed, partially lit and not pitch black.

If it is required to animate a mesh and make it move, we have to define an armature which is made of a series of invisible bones connected to each other via parenting or constraints, that allow we to pose and deform the geometry that surrounds it. The armature is used for building skeletal systems to animate the poses of characters and everything else which needs to be posed. By adding an armature system to an object, it can be deformed accurately so that geometry doesn't have to be animated "manually". The armature modifier allows objects to be deformed simply by specifying the name of the virtual bone. As a bone moves, it deforms or moves the vertices, but not necessarily all of them, only the ones assigned to it. The mesh surface is analogous to the skin of the human body. The armature is al so referred as Skel eton. In some complex 3D programs there are more complex skeleton systems defined bipeds. These elements are pre-designed and are customizable in order to fit with the mesh. An example of biped is in Fig. 9. 


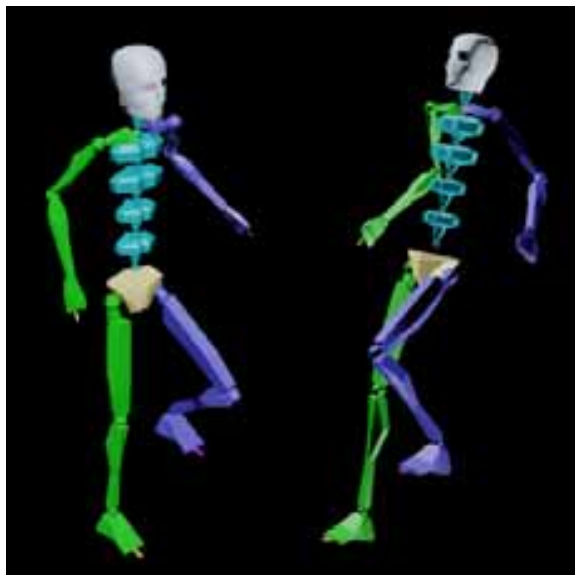

Fig. 9. Biped: a complex customizable skeleton system

By the 3D software an user can define how many fingers each hand has and how many phalanxes each finger has or how many sections for the torso and so on. Then the virtual representation of a real element (an object, a person or whatever) can be defined as a virtual alter-ego immersed in the virtual space.

In order to represent a human character we need to create a 3D body model, more often called A vatar (see Fig. 10). In a real-time virtual environment an avatar is a textured mesh obviously rigged, with a skeleton inside as reported.

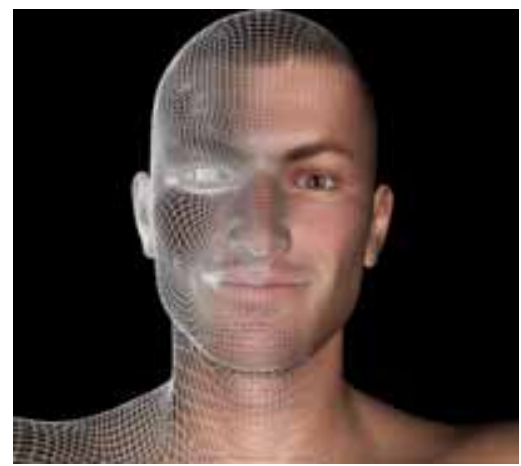

Fig. 10. 3D male avatar

There are many ways of creating a 3D model: starting from zero with a software for graphical applications by modeling a solid, as a sculptor would, or by editing an existing base model, or by creating a new one with external tools like 3D scanners for surface or volume acquisition. In any case we have to use a 3D graphic software to edit one or more meshes in a virtual complete environment, called Scene. There are several interesting software, freeware or under license, for such a purpose, and the best choice cannot necessarily correspond to the highest price. Usually the most expensive software is also too complicate to be utilized by beginner users. Among 3D design packages with commercial license, the most common and popular are 3D S Studio M ax, M aya and Lightwave 3D. Two of 
the best freeware solutions are DA Z 3D and Blender. The latter is not only free of charge but even open source and with a wide available toolset, defining a complete pipeline (from modeling to sequence editing) controlled by a flexible and consistent user interface. With Blender it is possible to create a scene that is a way to organize the 3D environment with objects, textures and lights. Each scene can contain multiple objects, which can contain multiple materials, which can contain many textures, and so on.

A 3D scene is like a real space with its own coordinate system. In a 3D cartesian coordinate system, a generic point is referred to by three real numbers (the coordinates), indicating the positions of the perpendicular projections from the point to three fixed, perpendicular, graduated lines, called the axes, which intersect at the origin. Stands the several possible choices among softwares, unfortunately it has not be defined a unique coordinate reference set yet. 3D graphics applications use two types of Cartesian coordinate systems: righthanded (Fig. 11B), and left-handed (Fig. 11A).
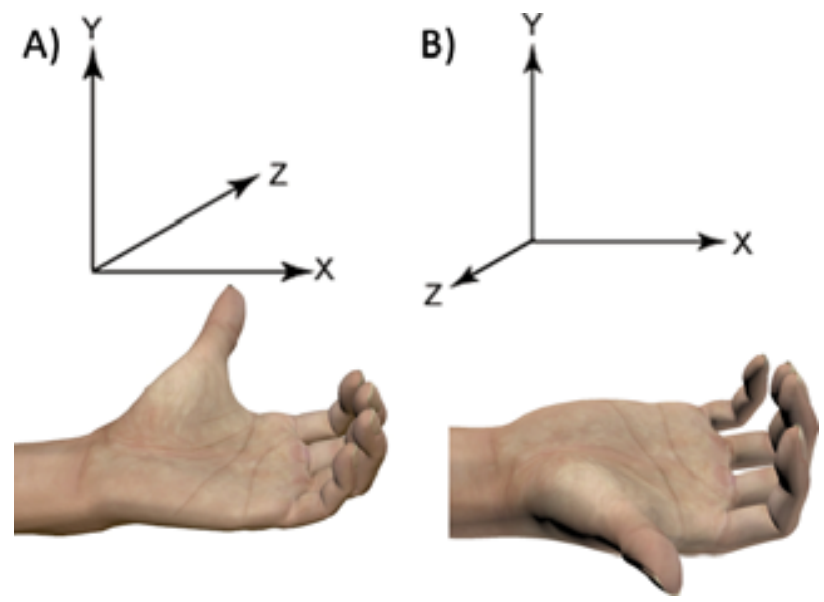

Fig. 11. A) left handed and B) right handed coordinate systems.

In both coordinate systems, the positive x-axis points to the right, and the positive y-axis points up. It is possible to remember which direction the positive z-axis points by pointing the fingers of either left or right hand in the positive $x$-direction and curling them into the positive y-direction. The thumb points in the positive z-axis direction for that coordinate system.

The basic operations performed on objects, defined in a 3D coordinate system, are rotation, translation and scaling. It is possible to combine these basic transformations to create a transform matrix, as it will be detailed explained in the next section. Likewise our eyes see the reality, in the virtual reality the scene is observed by virtual cameras. Just like the corresponding real cameras, the virtual ones may be located in a three-dimensional space, capturing the scene from their point of view. Everything located inside the virtual camera field of view is called view port.

\subsection{Reality-virtuality interactions}

The previous section deals with the elements existing in a virtual artificial world in order to reproduce reality. This section is dedicated to specify how to import a real action or 
movement in a 3D virtual scene and consequently how reality and virtuality can interact and the tools devoted to this aim.

As a starting point we need to define the medium of interaction between the real and the virtual world. In this sense we can utilize several interfaces such as mouse, touchpad, keyboard, joystick and so on, but the most interesting ones are the devices that allow users to move naturally within the environment, giving them an "immersive" experience. These systems can be based on webcams, capable to track human movements, or can be based on motion sensors directly sewn on garments (as is the so called data glove detailed afterwards).

Referring the sensors as the key elements capable to reveal the states of the (active or passive) sources, the commercial human movement tracking systems fit basically into three different classifications depending on where the sensors and the sources are respectively placed (Wang, 2005):

Inside-O ut Systems: the sensors are positioned on the body while the sources are somewhere elsein the world. The problem with these systems is that they tend to be bulky.

O utside-In Systems: the sources are attached to the body while the sensors are somewhere else in the world. These systems are less intrusive to the subject but are particularly sensitive to occlusion problems.

Inside.In Systems: the sensors and sources are on the user's body. These systems can be used to study relative movements between specific parts of the body.

Obviously each system exhibits both advantages and disadvantages: to increase accuracy and to reduce latency the best choice is an Inside-In System (like wearable devices can be). On the other hand, a good choice to obtain an intrusion decrease, is an Outside-In System.

As an example, let's consider the acquisition of the human hand movement: the first issue is to find all degrees of freedom, taking into account that for each finger's joint not all movements are possible. The distal interphalangeal joint and the proximal interphalangeal joint have only the possibility to move in the flexion/ extension plane (Fig. 12C), so 1 Degree of Freedom ( 1 DoF). The metacarpo phalangeal joint has 2 DoF: one on the flexion/ extension plane and the other one on abduction/ adduction plane (Fig. 12d). The first thumb joint and the wrist have 3 DoFs and 2 DoFs respectively.

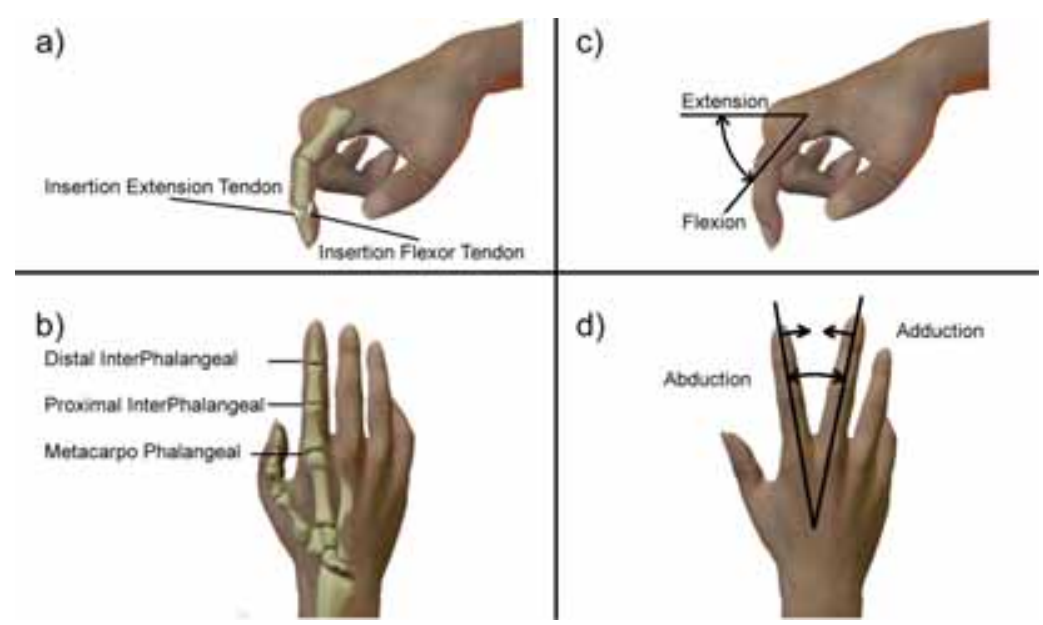

Fig. 12. a) and b) details of finger joints. c) and d) possiblemovements. 
After DoFs have been identified, we can measure all of them or restrict the interest to the ones which are strictly necessary to our aims and, consequently, focus on the subset of sensors, necessary to measure the requested DoFs.

After the most suitable acquisition system has been chosen and an appropriate 3D reproduction software has been selected, then it is possible to reproduce every recorded movements in a 3D virtual space with a high and realistic immersion degree.

A data glove, as for instance our Hiteg-Glove (http:/ / hiteg.uniroma2.it), is an example of Insideln System. Thanks to this wearable device it is possible to track real hand's movements ( 1 sensor for each DoF), and converting them into electric signals. Once data are acquired and converted into digital form, all values are sent to PC with a specific protocol useful to disambiguate and recognize the exact sensor under investigation and its value. The data can be tidily stored in a specific database, in such a way each information can be simply re-called and utilized in a simple numerical format or, more effectively, converted into a graphical representation useful for replicating the real hand movement by a virtual avatar on a PC screen. The software converts the digital values into bending values, expressed in degrees or radians, applying such bending to the corresponding virtual model.

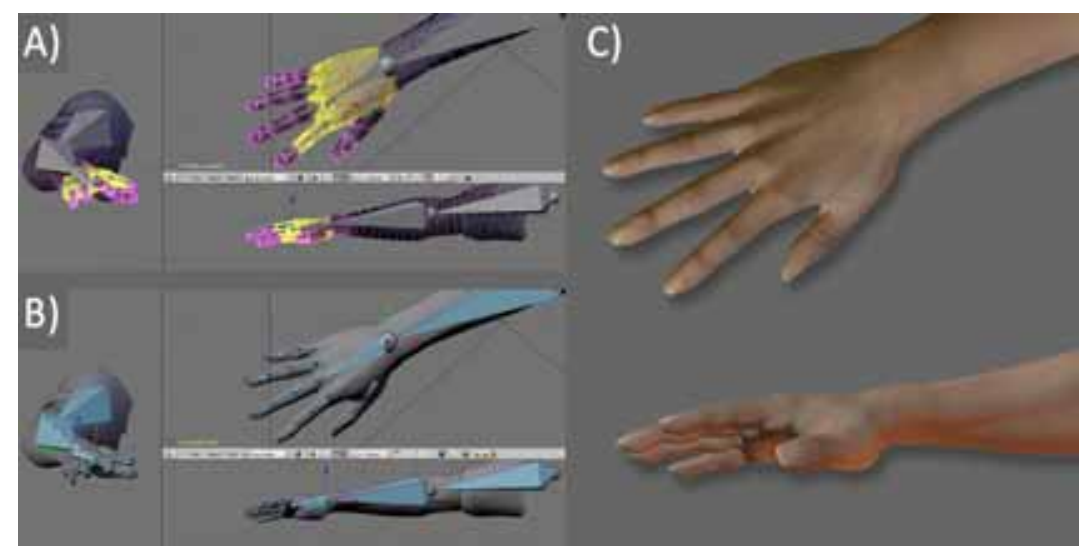

Fig. 13. A 3D human hand model: A) Mesh with vertex group (yellow selection). B)

A rmature: hidden hand bones. C) Final rendering of the rigged model with textures and lights

In a vectorial virtual space every bone is described by two matrixes: a local one (local transform) and a combined one (combined transform). The local matrix describes translations and rotations with respect to its pivot. Obviously the bones are connected in a way that translation and rotation of one bone influences the others in a cascading way. This means that for every junction movement the combined matrix of all the junctions is recomputed in a recursive way:

$$
C_{i}=L_{i} C_{i-1}
$$

The combined matrix of the $\mathrm{i}$-th junction $\left(\mathrm{C}_{\mathrm{i}}\right)$ is computed multiplying its local matrix $\left(\mathrm{L}_{\mathrm{i}}\right)$ by the combined matrix of the "father junction" $\left(C_{i-1}\right)$.

By a constant application of these transformations, the virtual model is consistent with the movements and the postures of the user. 
Once it has been established the way to move the mesh in the virtual world, it is necessary to make it interact with the objects or other animated meshes (other avatars) and in general there are two ways to do it. The first way is to recognize the posture or the action the user is making and to start a pre-set animation, if the action is known and allowed in that context. For example, the user indicates or touches an object in the virtual world and the software moves the object of a default measure, not related to real strength, speed or angle of the user. The second, more complex, way is to introduce a "physical engine" in the virtual world. A physical engine is a computer program that simulates Newtonian physic models using variables such as mass, velocity, friction, etc.. So it is possible to obtain simulated occurrences of collisions, rebounds, trajectories. With this engine, each object interacts with the surroundings according to its own characteristics.

We developed an interesting application just taking advantages of the virtual interaction possibilities in the virtual world. Actually one of the project carried on by our group named Hiteg (Health Involved Technical Engineering Group) is oriented to virtual architecture: the aim is to reconstruct entire monuments starting from pieces of ruins acquired by 3D scanner (see Fig. 14 and Fig. 15).

In such a way the real reconstruction can be made only after it is well known the exact location for every single pieces, so saving even a huge amount of money and time.

So far we have seen how you can act in the virtual world, but actually in reality the interaction is bidirectional: the world also affects the person. In this case it is necessary to use an haptic device.

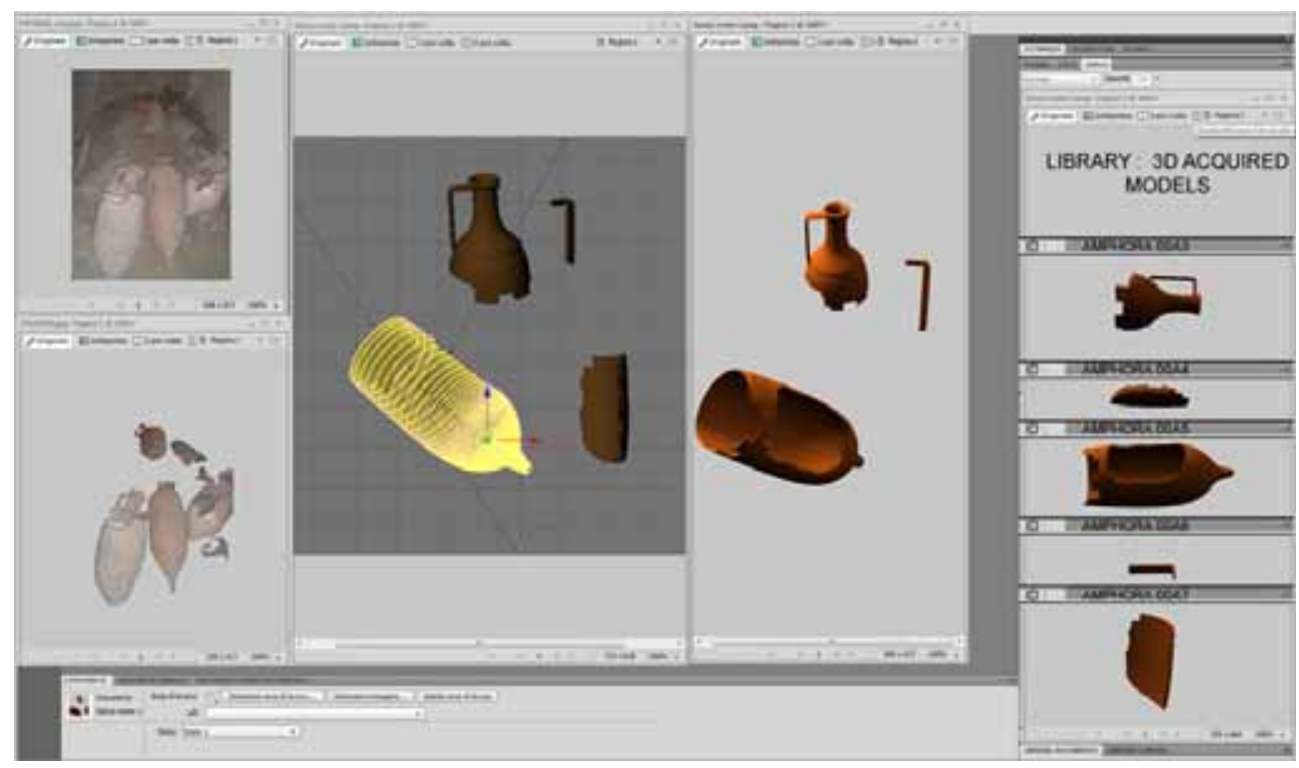

Fig. 14. The 3D scanned parts of an ancient pillar are virtually reproduced in a VR environment. 


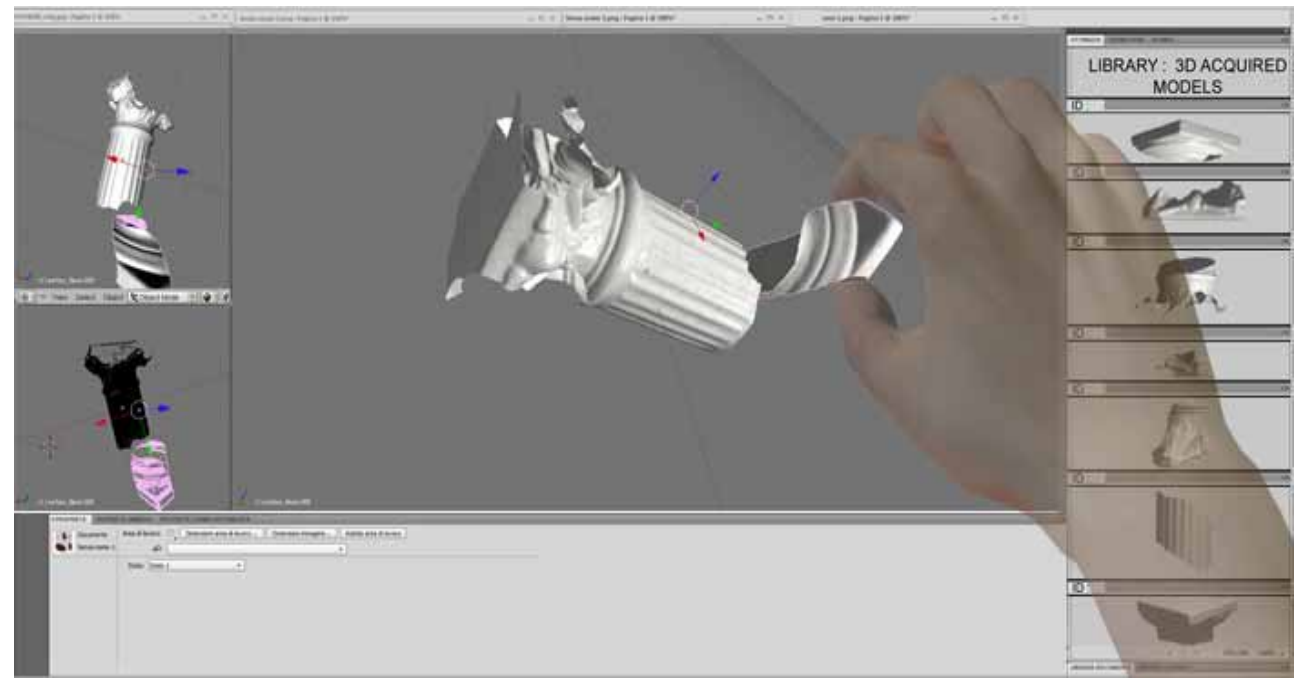

Fig. 15. 3D scanned parts of an ancient pillar are virtually recombined into the original form utilizing our Hiteg-glove as an interaction tool

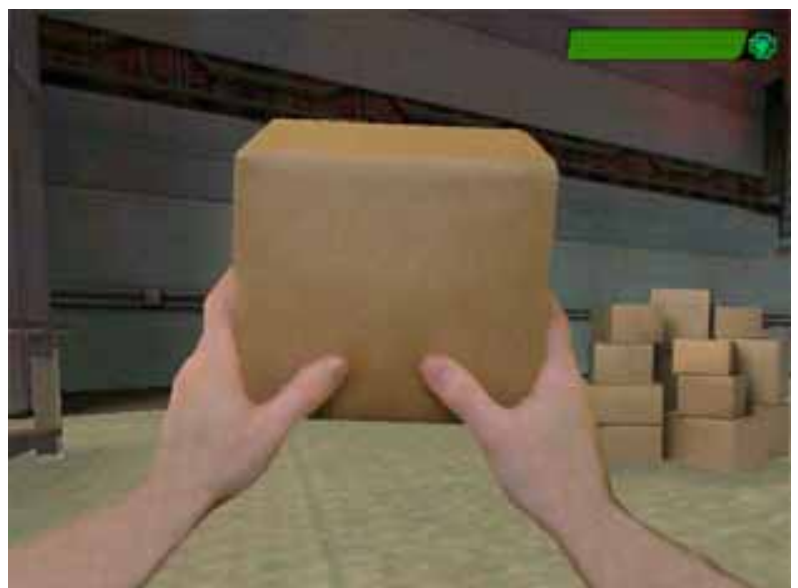

Fig. 16. When an object is virtually touched, the haptic device can give the sensation of the real touch.

The word haptic comes from the Greek verb ánтıкós, haptes, with the meaning of "contact" or "touch". Haptic technology, or haptics, is a tactile feedback technology that applies forces, vibrations, and/ or motions to the user. This mechanical stimulation may be used to assist the user in the remote control of machines and devices (tele-operations).

Now we have a world as close as possible to reality, which enjoys some of the fundamental laws of physics, we have an avatar, that represents the user, and a set of objects that enrich the environment. 


\subsection{Hiteg glove}

Generally speaking, a data glove is intended as the ensemble of mechanical to electrical transducers, a support (usually Lycra based), a powered conditioning electronic, a (wired or wireless) transmission system, all useful to measure all the DoFs of wrist and finger joints.

In our laboratory we developed a version of data glove, referred as HITEG glove (stands our name "Health Involved Technical Engineering Group"), which is mostly based on bend sensors capable of measuring bending angles thanks to the piezoresistive effect by means of which, in correspondence of each angle to which the sensors are subjected, it is measured a distinct resistance value.

In order to obtain high level performances of our data glove, in the sense of reliability, reproducibility and sensibility, we measured and characterized several piezoelectric sensors, manufactured by Flexpoint Sensor System Inc. (www.flexpoint.com) and Image S.I. (www.imagesco.com), different in length and encapsulation materials.

Sensors resistance variation vs. bending angle was measured thanks to a home-made setup based on hinges where the sensors lay on, and a stepper motor which provides the rotation of one wing of the hinge (with respect to the other which is fix constrained) simulating a human finger joint rotation (seeFig. 17).

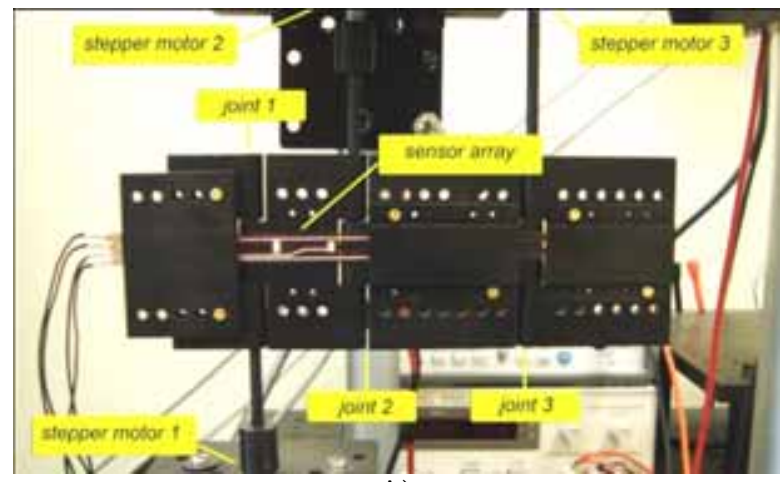

A)

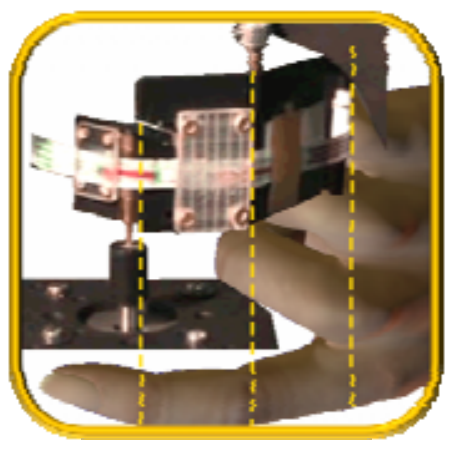

B)

Fig. 17. A) A three motor system to measure a sensor array, simulating movements of B) a real human finger

The motor which rotates the hinge's wing is a Trinamic PD-109 two phase hybrid stepper, microstepping optimized. It is provided with a Trinamic Motion Control Language (TMCL) which consists of an instruction set of motion control commands. On the basis of a host computer PC software development environment, the TMCL-IDE, motion control commands are provided to the motors. A rigid frame provides the necessary stability to the system. The motor is fixed on an optical bench by angular Newport EQ80-E shores. The motor motion is transmitted to the hinge's axis thanks to an universal rigid joint in order to obtain an excellent stability.

With the described measurement set-up, each sensor can be characterized in a $-90^{\circ}$ to $180^{\circ}$ (from inward to outward) range with programmable step value of bending angle, number of measurement repetitions and mechanical actuator speed. At known angles, the resistance values of the sensors are measured by an Agilent 34405A multimeter. 
The investigated piezoelectric sensors or array of sensors, for the specific application of the data glove, have a large measurement range for chosen outward angles from $0^{\circ}$ to $120^{\circ}$, and correspondingly the resistance normally changes from 10 to $170 \mathrm{k} \Omega$. The hysteresis of the sensors is really negligible and measurement repeatability is exceptional. Among all the performed measurements, some relevant results are showed in Fig. 18. It reports measurement results, resistance mean values (including standard deviations), on 6 different 2 inches length polyimmide encapsulated Flexpoint sample sensors: each sensor is characterized repeating measurements 10 times, varying bending angle from $0^{\circ}$ to $120^{\circ}$ and vice versa.

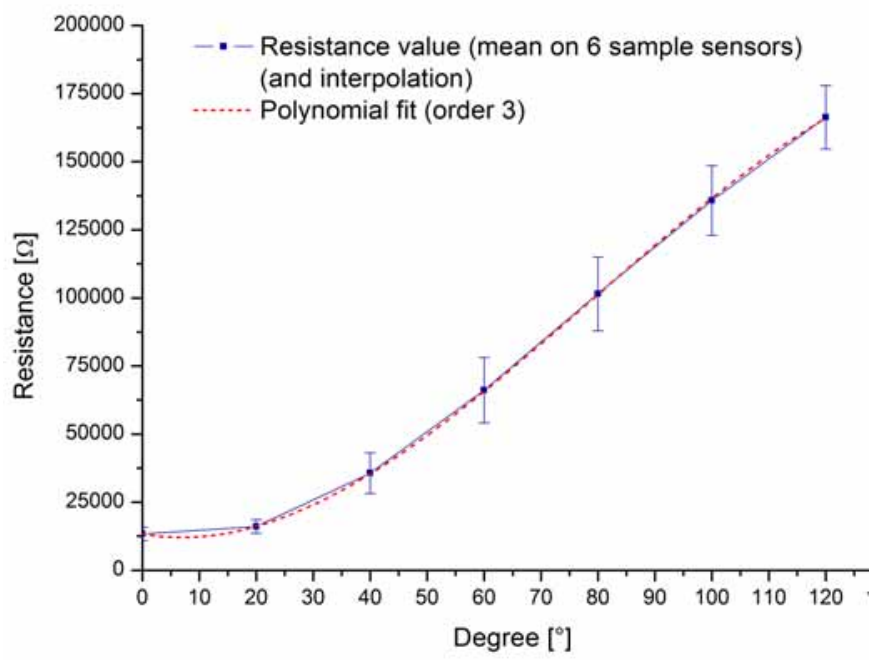

Fig. 18. Resistance variation vs. bending angle: mean on 6 sample sensors and standard deviation

A fter the characterization, the sensors are sewn on a Lycra based glove, each corresponding to a single finger joint so to measure flex-extension movements and between fingers to evaluate abdu-adduction movements. The Fig. 19 shows our Hiteg glove.

The resistance variation vs. bending angle characteristic of each sensors is utilized to correctly convert the electric resistance value into the corresponding finger joint flexed angle.

\section{E-health applications}

Healthcare is one of the areas potentially dramatically reshaped by the introduction of the new virtual tools. In fact, over the past few years there has been a rapid increase in the application of VR technology for e-heal th purposes in particular in diagnosis, healing, motor and neurological rehabilitation, motor therapy, instruction and surgery simulation, study, even in explanation to patients. With the aim of VR in motor therapy doctors have a multiview vision so to better catch pathologies (Saggio et al., 2009), in rehabilitation to quantitatively, not qualitatively, eval uate pre-post effect of surgery (Castagnaro et al., 2010), in functional analysis so to create a database to classify the residual movement of the 


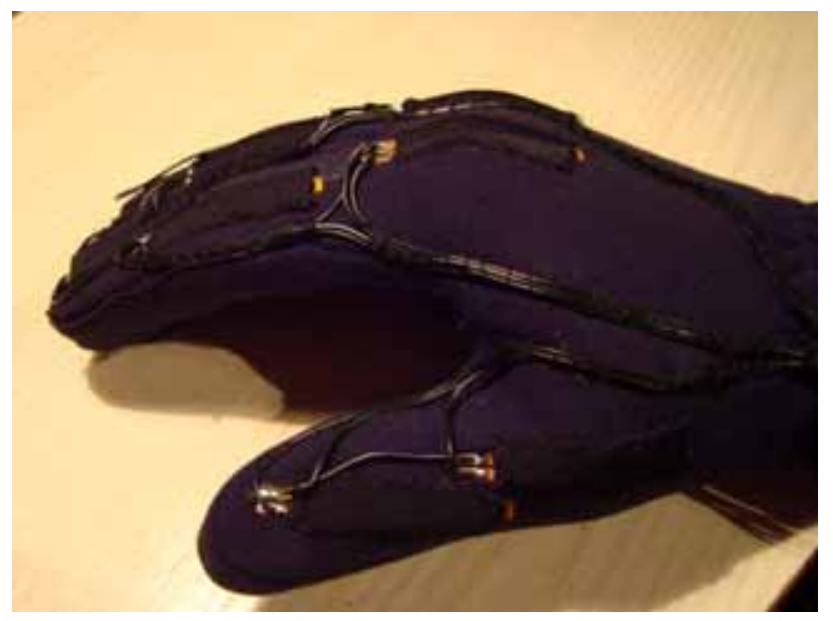

Fig. 19. The Hiteg glove

patient, in doctor training so to evaluate their surgical skills, in understanding the genesis and treatment of special symptoms (Zányi et al., 2009), in biomechanical evaluation during work and daily-life activities (Draicchio et al., 2010), in pain reduction treatments (Shahrbanian et al., 2009), even in developing an additional therapy tool for eating and impulse control disorders (Jiménez-Murcia et al., 2009), etc.

In the following paragraphs, we will focus our attention on some e-health applications of VR. In particular we will detail of surgery simulations, neurological and motor rehabilitations, brain computer interface facilities for severe neuromuscular impaired people.

\subsection{Surgery simulator}

Surgery simulator is an excellent example of application of virtuality and reality-virtuality interaction. Differing from classic computer graphics, with the surgery simulator the user can touch and interact with objects such as organs or human tissue.

The first historical simulator was invented by Edwin Albert Link in 1929. It was a flight simulator called the "Blue Box" or "Link Trainer" by which it was possible to evaluated the pilot performances "off-line". Many years passed since that date before the idea could be applied in medicine, but in the latest years it is having an increasing implementation. Just to refer the importance of the surgery simulator for the market, looking at the U.S. market for instance, the surgical training systems sold had a value of approximately 9.9M \$ in 2004, over $11 \mathrm{M} \$$ in 2005 , till more than $26 \mathrm{M} \$$ in 2009 . It is believed that worldwide sales more than double U.S. sales. But there is not only a market for the surgery simulator itself: the system providers can generate percentage on the revenue by providing updates to software, training, and system support.

The surgery simulator is more and more becoming an important, or somewhat fundamental, tool useful to train novice surgeons to practice complex operative tasks before entering the operating room. To practice a procedure or a given gesture repeatedly can dramatically increase surgical training, so surgery simulator can even become useful for those who are already practice but want to gain greater proficiency outside the operating room. It is 
somewhat ascertained how simulators are helping to accelerate that learning curve, so improving the fundamental Patient Safety (www.oregonsimulation.com, www.surgicalscience.com). Using VR concepts (navigation, interaction, immersion), surgical planning, training, and teaching even for complex surgical procedure may be possible. A hypothesis regards even visionary leaders examining the VR as an educational technique integrated into traditional curricula of novice surgeons (Dawson, 2006).

At the end, the simulator can record errors and provide an operative efficiency measure, so functioning both as an educational tool and a skills validation instrument.

N owadays VR is exploited in some specific surgery simulator applications: hepatic surgery (Marescaux et al., 1998), arthroscopy trainer (www.insightarthrovr.com), laparoscopic surgery (Ho, 2006; Schijven \& Jakimowicz, 2003), endoscopic procedures (Soler et al., 2009), virtual cholecystectomy (www.surgical-science.com), thoracic surgery, orthopedics, urology, and gynecology (Haluck et al., 2002), arterial/ duct clipping (Chaudhry et al., 1999), endovascular procedures (Van Herzeele \& Aggarwal, 2008) and so on, but with time it is reasonable to think that the real most part of surgery will be computer simulated.

Since the peculiar application, for which virtual environments are interactive and reactive, the interactions which can occur are of unpredictable nature, so it is not possible to pre compute images for each of the 20 frames/ $\mathrm{s}$ that are needed to provide an immersive VR experience. As if not enough, the physical correct behaviour of objects have to be modelled in real-time too. So, in practice, at present there is a limit to the desired realism of the physical models, and it must be balanced against the need for speed. In any case there are interesting studies and applications which demonstrate surgery simulations with an acceptable real-time occurrences. Since now modelling deformation in virtual anatomy has been realized by using surface models (Cover et al., 1993), but more recently the volumetric mass-spring models (Kuhn, et al., 1996), the Finite Element models (Bro-Nielsen \& Cotin, 1996), and the Fast Finite Element models (Bro-N ielsen, 1996), demonstrate a 3D volumetric deformable patient organs in a more than acceptable real-time modelling.

The other key element is the possibility for a doctor to have a force feedback to his/ her movements/ action. In fact to correctly act, it can be fundamental to have in his/ her hands a force feedback that mimics, for instance, how tissue and blood vessels feel and behave or heart beats in real life. A dissertation on methods and fundamental considerations for adding force feedback to a surgery simulator has been reported elsewhere, where an example of the virtual endoscopic surgery trainer "VS-One", developed at the Forschungszentrum Karlsruhe, is treated (Maass et al., 2003).

\subsection{Virtual Reality in rehabilitation}

Virtual Reality in rehabilitation treatments is becoming more and more applied. The new VR technologies can potentiality improve the dynamic posturography for a better understanding of standing balance in clinical settings, may improve gait for people with amputations, can visualize how ultrasound and laser treatments may benefit wound healing, and so on. Within all the possibilities, we will focus our discussion on some of the most important rehabilitation treatments, especially respect the cognitive/ neurological and postural training/ motor rehabilitation.

\section{Cognitive and N eurological Rehabilitation}

Only in the U.S., traumatic brain injuries (TBI) resulting from car crashes, falls, gunshot wounds, and sports injuries is 500,000 to $1.9 \mathrm{M}$ persons (Rizzo et al., 1998), and accounts 
around 200,000 hospitalized cases per year. Over $1.7 \mathrm{M}$ persons suffer a mild TBI that results in a temporary disability (Torner et al., 1999), while 100,000 people suffer varying degrees of permanent disability from TBI. So, it becomes obvious the importance of the following rehabilitation which can greatly contribute to reduce impairment, disability and handicap (Rose, 1996). Since it has been shown that the activity of mental stimulation induced by virtual reality can change the brain metabolic activity, it follows that exercising in a virtual environment offers the potential for significant gains in cognitive function (Grealy et al., 1999) greatly improve the rehabilitation process.

In order to achieve restoration of cognitive functions, a subject must repetitively perform appropriate retraining exercises. In this view, VR can help people with brain injuries to regain the ability to do simple activities by retraining damaged brain areas or enabling patients to learn to use new areas. VR can also prepare people who have lost some sensory functions for navigation in an unfamiliar place by letting them first experience a virtual (e.g. audio and haptic) layout of that setting. The fully advantages of VR technology for rehabilitation of the activities of daily living has been reported (Lee et al., 2003). Among the advantages of adopting the VR technique, is the fact that it allows to easily vary training parameters and to explore especially effective scenes that may be difficult or unsafe to construct in the real world.

The VR can be not necessary intended as a substitute of standard techniques useful for rehabilitation abilities after brain damage, but novel and standard methods often strictly coexist together being applied in a parallel or series way (Koenig, 2009). In addiction to VR, also mixed reality demonstrates its efficacy for the assessment of post-traumatic stress disorder with (or without) TBI (Fidopiastis et al., 2009).

Postural training and motor rehabilitation

Visual, vestibular, and proprioceptive sensory information play a key role in postural and motor effectiveness, and the integration of multisensory information from the environment is the basis for the control of body spatial orientation and movement (Cinelli \& Patla, 2008). On the other end, the adoption of postural measurements and VR can allow new paradigms aimed at altering the multisensory information contribution thus opening up many new research possibilities. These arguments imply that the tools offered by VR can be dangerous or can produce benefits in postural training and motor rehabilitation (Menegoni, 2009), so particular attention must be paid and ad-hoc protocols must be carefully developed (Boechler et al., 2009; Trotti et al., 2009).

Several requirements and long time repetitive practices are necessary for effective postural training and motor rehabilitation intervention. But the average situation for hospitalized stroke patients is that they receive half an hour session daily by a therapist, so the healing time is often too long. The use of the VR technology can drastically reduce this problem by allowing patients to perform long time and high frequency rehabilitation exercises using a computer, even simply at home. Last frontier in this field is the Haptic Motor Rehabilitation since it offers force and tactile feedback which can be crucial for many upper and lower extremity rehabilitation (Kayyali et al., 2007).

A recent study demonstrates how virtual environments and VR can offer, if correctly adopted, a valid tool in motor rehabilitation with respect those achieved in real-world applications (Sveistrup, 2004).

So, many efforts have been paid to develop valid systems based on VR. As an example, the N euroVR 1.5 is a cost-free virtual reality platform based on open-source components, 
allowing professionals to easily modify a virtual world, to best suit the needs of the clinical setting (Algeri et al., 2009).

By our side, we developed a complete VR system specific for the upper limb motor rehabilitation, which is now being tested on hand injured patients at the Hospital structure of the ASL Viterbo (Italy), Hand Surgery Dept., thanks to Dr. Antonio Castagnaro and Dr. A nna De Leo (Castagnaro et al., 2010). The overall system consists of a wearable sensorized device (the data glove), an electronic circuitry for conditioning and A/D converting electrical signals, a wireless data transmission board based on ZigBee protocol, a database on SQL platform useful for data storage for further data utilization, a virtualization software capable to in-line and off-line reproduce the recorded movements by avatars (see a schematic representation in Figs. 20 and 21).

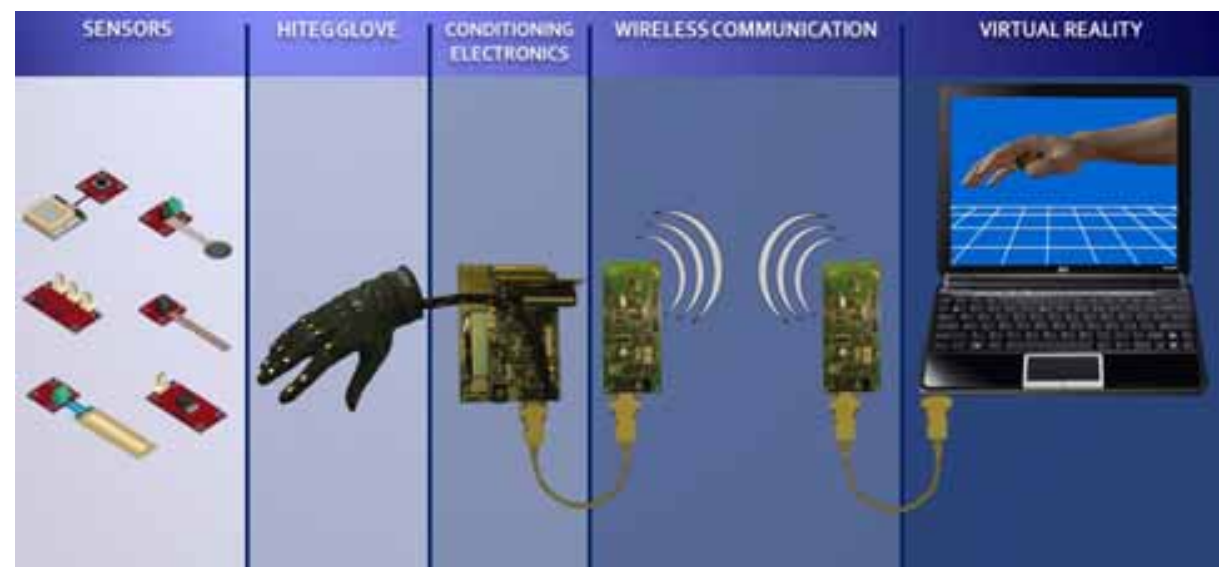

Fig. 20. A schematization of the overall system made of sensors, Hiteg glove, electronic for conditioning the signals, wireless communication board based on ZigBee protocol, and Virtual Reality reproduction

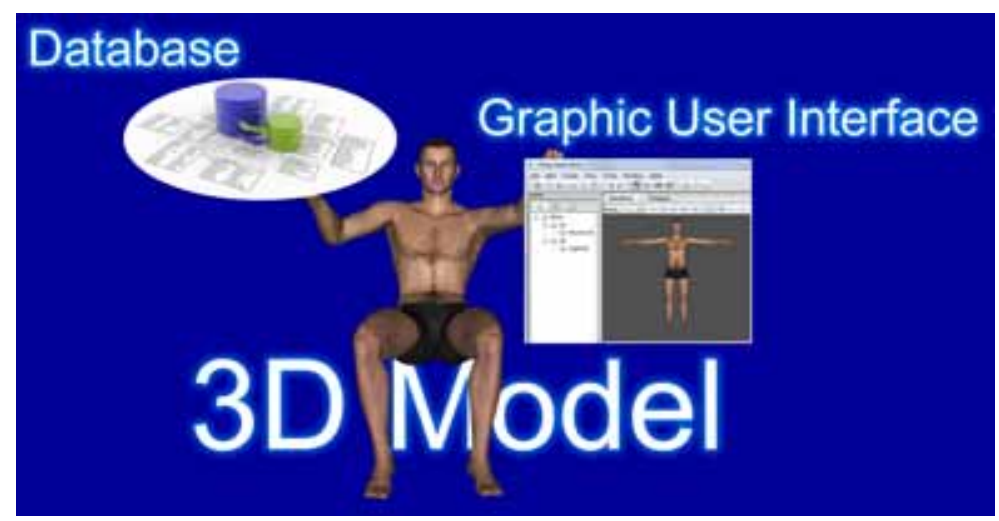

Fig. 21. A schematization of the VR system we adopted for motor rehabilitation. It is made of a 3D model, a database where recorded user movements are stored and a Graphic User Interface(GUI). 
During the post-processing data phase, thanks to the model, it is possible to replay all the fingers and hand movements in slow / rapid / frame-by-frame motion (see Fig. 22) and to isolate even just one finger at a time, removing the others from the view, in order to focus the operator's attention only on some important details.

The overall VR system is believed to become, in a near future, fully integrated to the surgery follow-up.

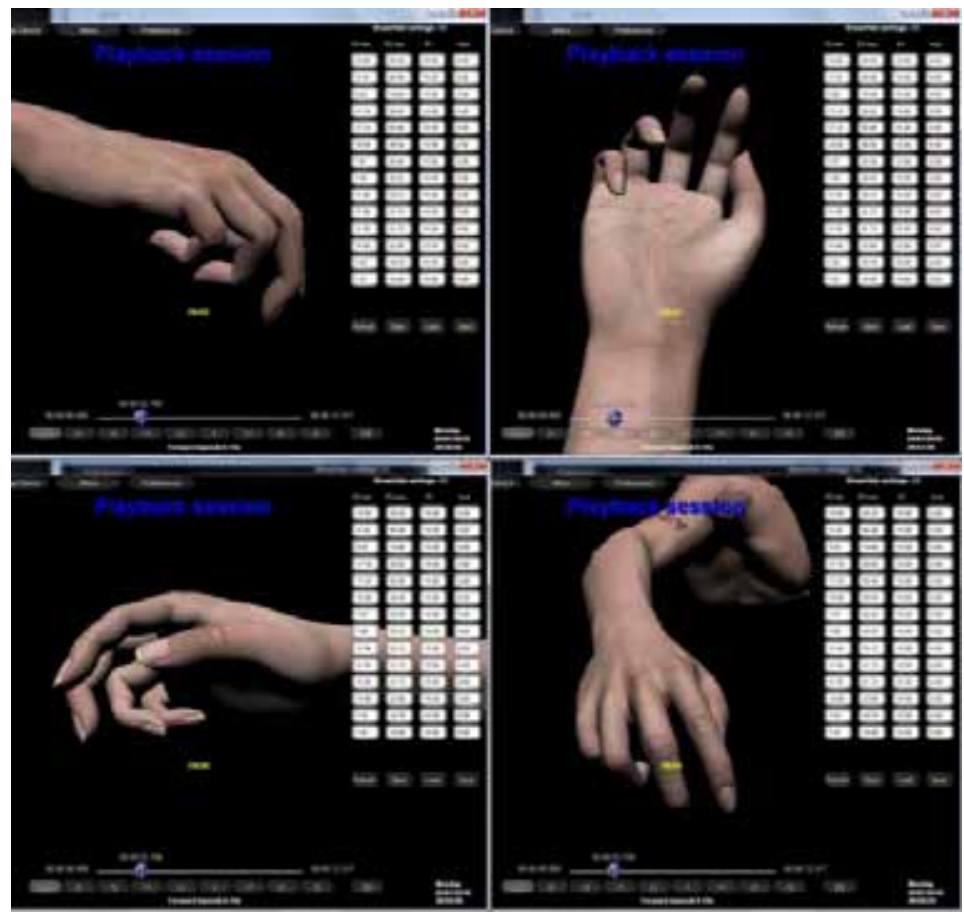

Fig. 22. A reproduction session: software allows user to see an acquisition session off-line, and by rotating 3D model in any direction, it is possible to analyze reproduction from different viewpoints.

\subsection{Brain Computer Interface}

Many people in the world are affected by severe neuromuscular impairments, which make them lose the control on their muscular voluntary activities thus isolating them from the environment. Brain Computer Interface (BCI) systems try to facilitate for these people the communication of their intents by translating some electrophysiological signals into commands towards external peripherals without making use of the classical pathways of nerves and muscles (Wolpaw et al. 2002). Basically BCl is adopted to assist and support impaired people. BCls bypass the user's peripheral nervous system (nerves) and his/ her muscles, establishing a direct connection between the central nervous system (brain) and the environment the user operates in. In this interaction paradigms, it is not needed that the user contracts even a single muscle (e.g. to press a button, to vocalize his/ her intent, or to direct his/ her gaze), because the interface is able to recognize specific commands by recognising his/ her "brain states" (Cincotti et al., 2009). 
There are different adopted methods to interface brain with computer, and these methods can be invasive (electrodes implanted directly into the gray matter of the brain, subdural Electro Cortico Graphy “ECoG", Stereotactic Electro Encephalo Graphy "SEEG",..) or non invasive (Electro Encephalo Graphy "EEG", Magnetic Resonance Image "MRI", Magneto Encephalo Graphy "MEG", functional Magnetic Resonance Imaging "fMRI" systems, ..).

One of the most fruitful areas of $\mathrm{BCl}$ research is the development of devices that can be controlled by thoughts, so giving to the severely disabled people the possibility to act independently.

Obviously the research look forward in adopting the less possible invasive interfacing technique, so particular attention is being paid to $\mathrm{BCl}$ systems essentially based on EEG recording (Costantini et al., 2009). But since the performance of $\mathrm{BCl}$ strictly depends on the ability of users to control and modulate their own EEG signals, it is of fundamental importance to develop a system capable to increase such a control. In this view studies emphasize how it is possible to improve the EEG control via feedback presentation (Bianchi et al., 2008). To get this objective, VR technology is becoming a powerful tool by its graphical possibilities to improve $\mathrm{BCl}$-feedback presentation, with the capability of creating immersive and motivating environments (Angevin, 2009).

Let's consider an user with upper limb immobility, or affected by a stroke with consequently motor decreasing. In this case, it can be useful to reproduce the hands of the user in a virtual environment and to stimulate his/ her motor imagery with a visual stimulus (like a spotlight on a specific arm). The user, connected to a $\mathrm{BCl}$ system, holds his/ her hands on a real desk; his/ her arms are hidden by a towel or a panel, on which a projector displays virtual hands reproduced as avatars. It is important that the 3D model is much similar as possible to the user real counterpart, in order to increase the realism and the positive response. For the same reason, it is very important that the perspective of the virtual model corresponds to the user's point of view. During a trial, the software creates some visual stimuli and records the EEG activity of the user. If the $\mathrm{BCI}$ system recognises the user's brain state, by means of classifiers (Saggio et al., 2010), then the avatars are animated so to reproduce the thought movement. A schematization of this feedback path is in Fig. 23, where the system provides as an output the movements of a robotic arm. At present, the overall system works thanks to a team we collaborate with, at the Santa Lucia IRCSS foundation in Rome, Italy.

\section{Conclusion}

N owadays the knowledge and understanding of the reality pass through the virtual world. This is practically true for all the human disciplines, but it is particularly true in e-health applications, for healing treatment, therapy, rehabilitation, surgery and support for impaired people. Since health is a widespread top priority, and will remain so in the context of an ageing society, it appears evident how every possible contribute to the increase of medical solutions and the decrease of time and cost of healing, are welcome. From this point of view VR is a really great opportunity within this challenge.

This chapter dealed with the techniques usefully adopted to convert the real into virtual and the advantages this passage offers having, in some way, assisted and/ or enriched the real world. We treated also of our and our collaborators efforts and results in designing, developing and applying 3D visualization techniques (stereoscopy and holography), human 


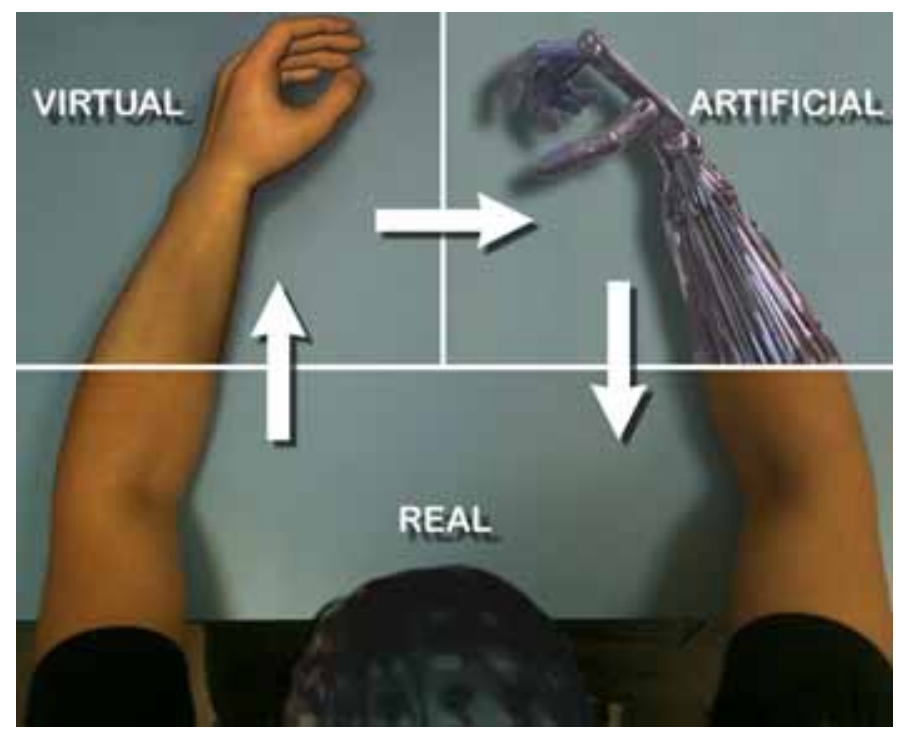

Fig. 23. The user wear a 61 electrodes cap by which his/ her mental status is recorded. When the $\mathrm{BCl}$ system recognizes a "closing hand" gesture, the virtual hand reproduces the gesture. The artificial limb can be driven as well.

postures measurements (Hiteg glove), avatar representation, human machine interaction (virtual mouse and keyboard), virtual interactions (reconstructions of ancient monuments), motor rehabilitation (for hand injured patients), communication for neuromuscular impaired people (BCl systems).

Special attention was paid to the basic processes necessary for reproducing reality in a virtual environment and the methodology for the reality-virtuality interactions. Some ehealth applications have been detailed, especially for surgery simulations and assisted rehabilitation processes.

We explained how the VR markets can be considered on the application areas of: rehabilitation and therapy (cognitive/ neurological, postural/ motor, pain distraction,...), surgery (simulations, pre-operative planning, assisted surgery), education and training (for novice surgeons, to practice procedures, to certify experienced surgeons,..), diagnostic tool also by means of visualization of medical data (2D, 3D modelling).

In conclusion we can state that, if correctly utilized, Virtual Reality can be a really valid support for e-health applications, especially because it can reduce time and cost for effective therapy/ treatment for health-focused disciplines, and provide doctors an opportunity to perform tasks in a risk-free environment. In addiction patient acceptance can be quite high since the VR implementation can havea low invasive impact.

\section{References}

Algeri D., Carelli L., Gaggioli A., Riva G. (2009). A Free Tool for Motor Rehabilitation: NeuroVR 1.5 with CamSpace. Annual Review of CyberTeraphy and Telemedicine, Brenda K. Wiederhold, Giuseppe Riva (Ed.), vol. 7, 2009, ISSN : 15548716, 99-101 
Angevin, R. R. (2009). Annual Review of CyberTeraphy and Telemedicine, Brenda K. Wiederhold, Giuseppe Riva (Ed.), vol. 7, 2009, ISSN : 15548716, 184-188

Azuma, Ronald T. (1997). A Survey of Augmented Reality, Teleoperators and Virtual Environments 6, 1997, 355-385, Malibu

Bianchi L., Pronestì D., A bbafati M., Quitadamo L. R., Marciani M. G., Saggio G. (2008). A New Visual Feed-Back Modality for the Reduction of Artifacts in Mu-Rhythm Based Brain-Computer Interfaces. 30th Annual International Conference of the IEEE Engineering in M edicine and Biology Society, EM BC, August 20-24, 2008, Vancouver, Canada

Boechler P., Krol A., Raso J. Blois T. (2009). Virtual Reality Interventions for Rehabilitation: Considerations for Developing Protocols. Annual Review of CyberTeraphy and Telemedicine, Brenda K. Wiederhold, Giuseppe Riva (Ed.), vol. 7, 2009, ISSN: 15548716, 102-104

Bro-Nielsen M. (1996). Surgery Simulation Using fast Finite Elements. Proceedings of the $4^{\text {th }}$ International Conference on V isualization in Biomedical Computing, 1996, 529 - 534

Bro-Nielsen M., Cotin S. (1996). Real-time volumetric deformable models for surgery simulation using finite elements and condensation. Computer Graphics Forum (Eurographics'96), vol. 15, no. 3, 1996, 57-66

Castagnaro A., De Leo A., Inciocchi S., Saggio G., Tarantino U. (2010). Nuova biotecnologia: il guanto sensorizzato per misurare la cinesi della mano. $48^{\circ}$ Congresso $\mathrm{N}$ azionale Società Italiana Chirurgia della M ano SICM 2010, "Stato dell'arte nella chirurgia articolare del gomito, polso e mano: protesi e tecniche alternative", 29 Sept - 2 Oct, Savona, Italy, 2010

Chaudhry A., Suttont C., Wood J., Stonet R., McCloy R. (1999). Learning rate for laparoscopic surgical skills on MIST VR, a virtual reality simulator: quality of human-computer interface. A nn R Coll Surg Engl., vol.81, 1999, 281-286

Cincotti F., Quitadamo L. R., A loise F., Bianchi L., Babiloni F., Mattia D. (2009). Interacting with the Environment through Non-invasive Brain-Computer Interfaces. In U niversal A ccess in H uman-Computer Interaction. Intelligent and U biquitous Interaction Environments, Springer Berlin / Heidelberg (Ed.), Vol. 5615, 2009, 483-492

Cinelli M. E., Patla A. E. (2008). Task-specific modulations of locomotor action parameters based on on-line visual information during collision avoidance with moving objects. H uman M ovement Science, vol. 27, 2008, 513-31.

Costantini G., Casali D., Carota M., Saggio G., Bianchi L., A bbafati M., Quitadamo L. R. (2009). SVM Classification of EEG Signals for Brain Computer Interface. W IR N '09; 19th Italian W orkshop on N eural N etworks; May 28-30, 2009, Sal erno, Italy

Costantini G., Todisco M., Saggio G. (2010). A Data Glove to Control a Real Time Granular Sound Synthesis Process. The International Multi-Conference on Complexity, Informatics and Cybernetics: IM CIC 2010, A pril 6th - 9th, 2010, Orlando, Florida USA

Cover S. A., Ezquerra N. F., O'Brien J. F., Rowe R., Gadacz T., Palm E. (1993). Interactively deformable models for surgery simulation. IEEE Computer Graphics \& A pplications, Nov. 1993, 68-75

Dawson S. (2006). Procedural Simulation: A Primer, Radiology, Vol. 241, Number 1 October 2006, 17-25 
Draicchio F., Silvetti A., Amici F., lavicoli S., Ranavolo A., Muscillo R., Schmid M., D'A lessio T., Sandrini G., Bartolo M., Orengo G., Saggio G., Conte C. (2010). Global Biomechanical Evaluation during Work and Daily-Life Activities. BIODEVICES International Conference on Biomedical Electronics and D evices: part of BIOSTEC The 3rd International Joint Conference on Biomedical Engineering Systems and Technologies, Valencia, Spain, 20-23 January 2010, 108-112

Fidopiastis C., Hughes C. E., Smith E.. (2009). Annual Review of CyberTeraphy and Telemedicine, Brenda K. Wiederhold, Giuseppe Riva (Ed.), vol. 7, 2009, ISSN: $15548716,216-220$

Geisen, S. (2005). Term paper - Augmented Reality in surgery, Seminar M edical Images, Universität Paderborn, Fakultät für Elektrotechnik/ Informatik / Mathematik

Grealy M., Johnson D., Rushton S. (1999). Improving cognitive function after brain injury: The use of exercise and virtual reality. Archives of Physical Medicine and Rehabilitation, Vol. 80, Issue 6, 1999, 661-667

Haluck R. S., Gallagher A. G., Satava R. M., Webster R., Bass T. L., Miller C. A. (2002). Reliability and validity of Endotower, a virtual reality trainer for angled endoscope navigation, Stud H ealth Technol Inform., vol.85, 2002, 179-84

Ho I. K. (2006). Laparoscopic Surgery. The A merican College of Gastroenterology, January 2006 Jiménez-Murcia S., Fernández-Aranda F., Kalapanidas E., Konstantas D., Ganchev T., Kocsis O., Lam T., Santamaría J. J., Raguin T., Breiteneder C., Kaufmann H., Davarakis C. (2009). Annual Review of CyberTeraphy and Telemedicine, Brenda K. Wiederhold, Giuseppe Riva (Ed.), vol. 7, 2009, ISSN : 15548716, 163-166

Kayyali R., Shirmohammadi S., El Saddik A., Lemaire E. (2007). Daily-Life Exercises for Haptic Motor Rehabilitation. HAVE 2007 - IEEE International Workshop on Haptic A udio Visual Environments and their A pplications, Ottawa - Canada, 12-14 October 2007, 118-123

Koenig S. T., Crucian G. P., Dalrymple-Alford J. C., Dünser A. (2009). Annual Review of CyberTeraphy and Telemedicine, Brenda K. Wiederhold, Giuseppe Riva (Ed.), vol. 7, 2009, ISSN : 15548716, 105-107

Kuhn Ch., Kuhnapfel U., Krumm H-G., Neisius B. (1996). A virtual reality based training system for minimally invasive surgery. Proceedings of Computer A ssisted Radiology (CAR' 96), 1996, 764-769

Lee J. H., Ku J., Cho W., Hahn W. Y., Kim In Y., Lee S.-M., Kang Y., Kim D. Y., Yu T., Wiederhold B. K., Wiederhold M. D., Kim Sun I. (2003). Virtual Reality System for the Assessment and Rehabilitation of the Activities of Daily Living. Cyberpsychology \& Behavior, Vol. 6, No. 4, 2003, 383-388

Maass H., Chantier B. B. A. , Çakmak H. K, Kühnapfel U. G. (2003). How to Add Force Feedback to a Surgery Simulator. Surgery Simulation and Soft Tissue M odeling. Vol. 2673, 2003, ISBN 978-3-540-40439-2

Marescaux J., Clement J-M., Tassetti V., Koehl C., Cotin S., Russier Y., Mutter D., Delingette H., Ayache N. (1998). Virtual Reality A pplied to Hepatic Surgery Simulation: The Next Revolution. A nnals of Surgery, Vol. 228, No. 5, 1998, 627-634 
Menegoni F., Albania G., Bigonia M., Priano L., Trotti C, Galli M., Mauro A. (2009). Walking in an Immersive Virtual Reality. A nnual Review of CyberTeraphy and Telemedicine, Brenda K. Wiederhold, Giuseppe Riva (Ed.), vol. 7, 2009, ISSN : 15548716, $72-76$

Rizzo, A., Buckwalter, J. G., Neumann, U. (1998). Basic issues in the application of virtual reality for the assessment and rehabilitation of cognitive impairments and functional disability. CyberPsychology \& Behavior, vol. 1, 1998, 59-78

Rose F. D.. (1996). Virtual reality in rehabilitation following traumatic brain injury. Proc. 1st Euro. Conf. Disability, V irtual Reality \& A ssoc. Tech., Maidenhead, UK, 1996, 5-12

Saggio G., Latessa G., De Santis F., Bianchi L., Quitadamo L. R., Marciani M. G., Giannini F. (2009). Virtual reality implementation as a useful software tool for e-health applications, $1^{\text {th }}$ IEEE International W OWM oM Workshop on Interdisciplinary Research on E-H ealth Services and Systems, IREHSS 2009, Kos, Greece

Saggio G., Cavallo P., Quitadamo L. R., Marciani M. G., Bianchi L., Costantini G., Susi G. (2010). Comparison of Different Classifiers on a reduced set of features for Mental Tasks-Based Brain-Computer Interface. BIOSIGNALS - International Conference on Bio-inspired Systems and Signal: part of BIOSTEC The 3rd International Joint Conference on Biomedical Engineering Systems and Technologies, Valencia, Spain, 20-23 January 2010, 174-179

Schijven M., Jakimowicz J. (2003). Virtual reality surgical Iaparoscopic simulators. Surgical Endoscopy (2003) 17: 1943-1950

Shahrbanian S., Ma X., Korner-Bitensky N., Simmonds M. J. (2009). Scientific Evidence for the Effectiveness of Virtual Reality for Pain Reduction in Adults with Acute or Chronic Pain. Annual Review of CyberTeraphy and Telemedicine, Brenda K. Wiederhold, Giuseppe Riva (Ed.), vol. 7, 2009, ISSN : 15548716, 40-43

Shuhaiber, Jeffrey H. (2004). Review article - Augmented Reality in Surgery. A rchives of Surgery, Vol. 139, Feb 2004, pp. 170-174

Soler L., Nicolau S., Hostettler A., Fasquel J-B., Agnus V., Charnoz A., Moreau J., Dallemagne B., Mutter D., Marescaux J. (2010). Virtual Reality and Augmented Reality applied to Endoscopic and notes procedures. W orld Congress on M edical Physics and Biomedical Engineering, 362-365, 2010, Springer Berlin Heidelberg, 978-3642-03905-8, Munich, Germany

Sveistrup H. (2004). Motor rehabilitation using virtual reality. Journal of $\mathrm{N}$ euroEngineering and Rehabilitation, 2004, 1-10

Torner J. C., Choi S., Barnes T. Y. (1999). Epidemiology of Head Injuries. Traumatic brain injury, Donald W. Marion (Ed.), 990-991, E. Thieme, 3-13-783402-3, New York

Trotti C., Menegoni F., Baudo S., Bigoni M., Galli M., Mauro A., (2009). Virtual Reality for the Upper Limb Motor Training in Stroke: A Case Report. Annual Review of CyberTeraphy and Telemedicine, Brenda K. Wiederhold, Giuseppe Riva (Ed.), vol. 7, 2009, ISSN : 15548716, 257-260

Van Herzeele I., Aggarwal R. (2008). Virtual Reality Simulation in the Endovascular Field. Interventional Cardiology, Touch Briefings 2008, pp. 41-45

Wang, Yifei. Human movement tracking using a wearable wireless sensor network. Master Thesis. Iowa State University, 2005. Ames, lowa 
Wolpaw J. R., Birbaumer N., McFarland D. J., Pfurtscheller G., Vaughan T. M. (2002). Braincomputer interfaces for communication and control. Clinical N europhysiology, 113(6), 2002, 767-791

Zányi E., Selmanovic E., Broome M., Czanner S., Birchwood M., Chalmers A., Singh S. (2009). Annual Review of CyberTeraphy and Telemedicine, Brenda K. Wiederhold, Giuseppe Riva (Ed.), vol. 7, 2009, ISSN : 15548716, 138-140 